\title{
Phase Transitions in Finite Nuclei and the Integer Nucleon Number Problem
}

\author{
R.F.Casten ${ }^{a}$, Dimitri Kusnezov ${ }^{b, \text {, and N.V.Zamfir }}{ }^{a, c, d}$ \\ ${ }^{a}$ WNSL, Yale University, New Haven, Connecticut 06520, USA \\ ${ }^{b}$ Center for Theoretical Physics, Yale University, New Haven, CT 06520-8120 \\ ${ }^{c}$ Clark University, Worcester, Massachusetts 01610, USA \\ ${ }^{d}$ National Institute for Physics and Nuclear Engineering, \\ Bucharest-Magurele, Romania
}

To Appear in Physical Review Letters, June 1999

\begin{abstract}
The study of spherical-deformed ground-state phase transitions in finite nuclei as a function of $\mathrm{N}$ and $\mathrm{Z}$ is hindered by the discrete values of the nucleon number. A resolution of the integer nucleon number problem, and evidence relating to phase transitions in finite nuclei, are discussed from the experimental point of view and interpreted within the framework of the interacting boson model.
\end{abstract}

\footnotetext{
${ }^{1}$ dimitri@nst4.physics.yale.edu
} 
It has been known for decades that nuclear properties change, often dramatically, as a function of $\mathrm{N}$ and $\mathrm{Z}$. However, the possibility of true nuclear phase coexistence and phase transitions in the evolution of nuclear structure as a function of nucleon number, in the sense of conventional condensed matter systems, has generally been discounted. The reason has to do with the finite nature of atomic nuclei and the fact that they contain integer numbers of nucleons. In order to discuss the concept of phase transitions [1] one needs to identify a control parameter as well as an order parameter. If the fluctuations in the order parameter are small, that is, if the data all lie along a compact trajectory as a function of the control parameter, the existence of a phase transition would be signaled by a critical point where the order parameter is discontinuous.

The difficulty in applying this approach to nuclei lies in the obvious fact that nuclei contain integer numbers of nucleons and, therefore, their properties change discretely as a function of $\mathrm{N}$ and $\mathrm{Z}$. This is illustrated in fig. 1a where a typical collective observable in even-even nuclei, the energy of the first $4^{+}$state, is plotted against neutron number for the $\operatorname{Sm}(\mathrm{Z}=62)$ isotopes. Clearly, an interesting (and well known) change in structure is occuring but the abscissa values are, by definition, discrete. Therefore, regardless of what the data do, the integer nucleon number requires that, at best, one can only connect adjacent points by straight line segments and one can only define differences in properties, not derivatives.

It is the purpose of this Letter to discuss phase transitions in finite nuclei and to suggest a resolution of the integer nucleon number problem. When one considers low energy nuclear structure, nuclear models offer the flexibility of having one or more continuous parameters that can serve as control parameters, allowing the study of critical phenomena. The situation in actual nuclei is different. In contrast to phase transitions in a specific nucleus, where excitation energy (or temperature) can be introduced as a control parameter, structure at low energy evolves from nucleus to nucleus as a function of $N$ and $Z$, which we have seen are not useful as control parameters. However, we will suggest an empirical quantity which is continuous and which does correlate extremely well the structural changes with $\mathrm{N}$ and $\mathrm{Z}$ across large regions of nuclei, and then apply this approach to the $\mathrm{A}=150$ region 3 , 4 , 5. Finally, a theoretical analysis with the Interacting Boson Model (IBM) [6] shows why sharp transition regions are indeed an expected feature of structural evolution.

We noted in fig. 1 that the data from a single element do not allow a discussion of phase transitional behavior. The data for collective nuclei in an entire region $(50<\mathrm{Z} \leq$ $66)$, shown in fig. 1b, only exacerbate the problem. The abscissa values remain discrete, but now another prerequisite for a phase transition disappears, namely, the absence of fluctuations. The scattering of the data obliterates any evidence of sharply discontinuous behavior.

How can we get around this situation in finite nuclei? A possible answer, which we propose here, is to choose a qualitatively different quantity to play the role of a control parameter, one that is at least potentially continuous. Of course, even if the abscissa points become continuously distributed, we need to produce an approach in which the fluctuations in the data are small. To do so, consider fig. 2a-c, which should be read as successive frames. In this figure, the same $\mathrm{E}\left(4_{1}^{+}\right)$data as in fig. 1 are plotted, not against 
$\mathrm{N}, \mathrm{Z}$, or $\mathrm{A}$, but against another collective observable, the energy, $\mathrm{E}\left(2_{1}^{+}\right)$, of the first $2^{+}$ state. The top left panel is no better for discussing phase transitional behavior than is fig. 1a. The data points are discrete both vertically and horizontally. Although a nucleus can, in principle, have any $2_{1}^{+}$energy, the nuclei (Sm isotopes) in fig. 2a have specific and discrete values since their properties change rapidly from isotope to isotope.

However, when the data for additional elements are added (fig. 2b,c) we see a behavior that is qualitatively different from fig. 1. The distribution of points as a function of $\mathrm{E}\left(2_{1}^{+}\right)$ successively fills in, yielding in fig. $2 \mathrm{c}$ a nearly continuous distribution. Next, we note that $\mathrm{E}\left(2_{1}^{+}\right)$clearly correlates nuclear equilibrium properties extraordinarily well. The data for different elements lie along essentially identical trajectories and thus the ensemble of data also lies along a single compact curve, with very small fluctuations. This thereby enables a discussion of the trajectory and a potential interpretation in terms of phase transitions. Of course, other observables that reflect the equilibrium configuration could also have been chosen. $\mathrm{E}\left(2_{1}^{+}\right)$is preferred, however, since it is well and accurately known in a large number of nuclei and is easy to measure in newly accessible nuclei.

Observables such as $\mathrm{E}\left(2_{1}^{+}\right), \mathrm{E}\left(4_{1}^{+}\right)$, separation energies and other measures of structure cannot, of course, rigorously speaking, be considered as control parameters since they are not independently variable as is, for example, the temperature in a condensed matter system. Nevertheless, $\mathrm{E}\left(2_{1}^{+}\right)$, de facto, plays a similar role to a control parameter, and fig. $2 \mathrm{c}$ suggests evidence for true phase transitional behavior as seen by the nearly discontinuous change in slope from 2.00 to 3.33 at a specific value of $\mathrm{E}\left(2_{1}^{+}\right)$which we denote $\mathrm{E}_{c}\left(2_{1}^{+}\right)$. The expanded view of the rotational region in fig. $2 \mathrm{~d}$ clearly shows the different slope for these nuclei. The change in slope occurs at $\mathrm{E}_{c}\left(2_{1}^{+}\right) \sim 120 \mathrm{keV}$, although this value may differ in regions where other shells are filling.

Figure 2c itself is not a new result. We have discussed the $\mathrm{E}\left(4_{1}^{+}\right)-\mathrm{E}\left(2_{1}^{+}\right)$and related correlations in ref. [7] and even broached the subject of phase transitional behavior. The correlation in fig. 2c has been discussed theoretically [8] in the context of the $1 / \mathrm{N}$ expansion method for the IBM, and extended 99, 10] empirically to observables for intrinsic excitations and to odd A and odd-odd nuclei. What is new here is the explicit discussion of the process of reaching fig. 2c as a way of resolving the finite nucleon number problem and identifying phase transitional behavior. Of course, since nuclei are finite systems, the phase transition is smoothed out over a narrow region of $2_{1}^{+}$energies.

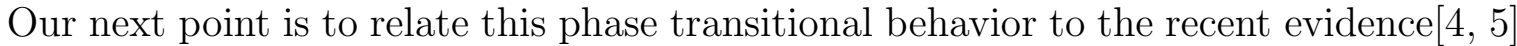
for phase coexistence in ${ }^{152} \mathrm{Sm}$ (not merely the familiar shape coexistence originating from an intruder state mechanism). Theoretical analysis 4 of ${ }^{152} \mathrm{Sm}$ points to two co-existing phases, a deformed ground state band and a spherical anharmonic vibrator spectrum built on the $0_{2}^{+}$level. Phase coexistence must occur at the critical point and so it is reassuring that the $2_{1}^{+}$energy of ${ }^{152} \mathrm{Sm}(121 \mathrm{keV})$ occurs at the same energy as that where the change in slope from 2.00 to 3.33 occurs in fig. 2c.

Thus, two different perspectives - phase coexistence in ${ }^{152} \mathrm{Sm}$ and the relation of yrast energies across the region of nuclei - give evidence for a phase transition from spherical to deformed structures, near $\mathrm{A}=150$. Note that, while phase coexistence occurs in a specific nucleus $\left({ }^{152} \mathrm{Sm}\right)$, the phase transition does not characterize a single nucleus, or the isotopes of an element, but is a property of, and only definable in terms of, an entire 
region. We stress here that this type of phase transition and phase coexistence is different from the shape coexistence picture known in other regions [11, 12]. Here the structural changes develop within the context of a single shell and do not involve an intruder state mechanism.

Other observables reflect these rapid structural changes. Two nucleon separation energies, $\mathrm{S}_{2 n}$, are sensitive to changes in equilibrium configurations. In fig. 3a we show the empirical values of $\mathrm{S}_{2 n}$ for the $50<\mathrm{Z} \leq 66$ nuclei (for all collective nuclei - those with $\mathrm{R}_{4 / 2}>2.05$ ). The $\mathrm{S}_{2 n}$ values have a well known, essentially parallel, shift in values for each successive Z. To compare values for different elements on the same scale, we therefore shift the separation energies for each $\mathrm{Z}$ by a constant amount chosen to give equal $\mathrm{S}_{2 n}$ values at $\mathrm{N}=88$ for the $\mathrm{N}>82$ shell and at $\mathrm{N}=76$ for the $\mathrm{N}<82$ shell.

For $\mathrm{N}>82$, the results for $\mathrm{S}_{2 n}$ in fig. 3a are as striking as for the $4_{1}^{+}$energies in fig. 2c. The behavior is compact, with small fluctuations and a sharp break in trajectory. This break occurs at a slightly lower $2_{1}^{+}$energy than the slope change for $\mathrm{E}\left(4_{1}^{+}\right)$in fig. 2c. Apparently, the $S_{2 n}$ values display a different dependence on the overall shape of the nuclear potential than low spin yrast levels (which presumably are most sensitive to the details of the potential near its minimum). For the lighter mass shell, $\mathrm{N}<82$, there are greater fluctuations and a gradual structural change but no evidence for a sharp phase transition.

In fig. $3 \mathrm{~b}$ we show $\mathrm{B}\left(\mathrm{E} 2: 2_{1}^{+} \rightarrow 0_{1}^{+}\right)$values as a function of $S_{2 n}$. The sudden break of trend at a specific value of $\mathrm{S}_{2 n}$ illustrates the point that $\mathrm{E}\left(4_{1}^{+}\right)$is not a unique measure of the structural transition: $B(E 2)$ values provide additional evidence for it.

The question arises as to why nuclei should behave in this way. As we have noted, it is easier to look at phase transitional behavior in a model since the model parameters are inherently continuous. Here, we consider the IBM since the analysis is simple but similar results characterize the Geometric Collective Model (GCM) [15]. We use the Extended CQF Hamiltonian[16, [17]

$$
H=\epsilon n_{d}-\kappa Q \cdot Q,
$$

where $Q=\left(s^{\dagger} \tilde{d}+d^{\dagger} s\right)+\chi\left(d^{\dagger} \tilde{d}\right)^{2}$, which has parameters $\epsilon, \kappa, \chi$ and the boson number $N_{B}$. Performing a systematic analysis of parameter space, IBM calculations of $E\left(4_{1}^{+}\right)$ versus $E\left(2_{1}^{+}\right)$reproduce the results of fig. 2c: they follow a slope of 2.00 above $\mathrm{E}_{c}\left(2_{1}^{+}\right)$for virtually any choice of $\epsilon, \chi$, and $\mathrm{N}_{B}$ that gives $\mathrm{R}_{4 / 2}=2.05-3.15$ as long as $\kappa$ is constant [18]. Indeed, the value of $\kappa$ determines the intercept $\epsilon_{4}$ [and $\mathrm{E}_{c}\left(2_{1}^{+}\right)$as well]. Were the data different from fig. 2c (e.g., scattered, or following a slope other than 2.00) the only way this behavior could be reproduced would be to separately adjust $\kappa$ for each nucleus, a clearly unlikely scenario, and one that is inconsistent with microscopic analyses of IBM parameters [19].

We now study the behavior of eq. (1) through the intrinsic state formalism[20, 21], computing the energy surfaces corresponding to different parameter values. It is convenient, for this purpose, to re-write eq. (1) with a control parameter $\xi=(1+\epsilon / \kappa)^{-1}$. The resulting scaled Hamiltonian has the form 


$$
H^{\prime}=(1-\xi) n_{d}-\xi Q \cdot Q
$$

For $\chi=-\sqrt{7} / 2,0 \leq \xi \leq 1$ maps the transition from $\mathrm{U}(5)$ to $\mathrm{SU}(3)$.

In fig. 4a we show the IBM energy surface corresponding to the classical limit of eq. (2), for $\mathrm{N}_{B}=10$ and $\chi=-\sqrt{7} / 2$, against $\xi$. The figure immediately shows the key point, that the location of the minimum in the energy, $\beta_{\text {min }}$, changes suddenly, for these parameters, at a particular $\xi$ value, from $\beta_{\text {min }}=0$ to a large finite value, as indicated by the dark line cutting through the contour plot. There are virtually no $\xi$ values for which intermediate $\beta_{\min }$ values result. This is consistent with the specific calculations in ref. [4. The IBM indicates that nuclei change abruptly from near spherical to deformed at a critical value $\xi_{\text {crit }}$. The evolution of the energy surface can be seen as the competition between two minima, spherical and deformed, rather than a gradual evolution from spherical to weakly deformed to large deformation. Such a level crossing scenario is, in fact, characteristic of a first order phase transition.

The qualitative behavior of these results is not sensitive to boson number $\mathrm{N}_{B}$ nor to variations in $\chi$. We show this in fig. $4 \mathrm{~b}$, which gives the values of the location of the lowest minimum of the energy surface, as a function of $\xi$ for a set of $\mathrm{N}_{B}$ and $\chi$ values. The curves all show the same behavior independent of the parameters: $\beta_{\min }$ is zero for small $\xi$ values, and then rises extremely rapidly to a saturation value within a very narrow range of $\xi$ values (which define a $\xi_{c r i t}$ for each $N_{B}$ and $\chi$ ).

In fig. $4 \mathrm{c}$, we show energy surfaces as a function of $\beta$ for IBM parameters applicable to each of the Sm isotopes. These surfaces range from near vibrator shapes for ${ }^{146,148} \mathrm{Sm}$ to softer in ${ }^{150} \mathrm{Sm}$, to the unique coexistence nucleus ${ }^{152} \mathrm{Sm}$ where two shallow minima occur, to ${ }^{154,156} \mathrm{Sm}$ where the prolate minimum is much deeper, thus localizing these latter nuclei at large positive $\beta$ values. The actual minimum in the energy surface occurs only for $\beta_{\text {min }} \sim 0$ or large positive $\beta_{\text {min }}$.

In summary, we have shown that finite nuclei can, and do, exhibit true phase transitional character by proposing a way to resolve the integer nucleon number problem. We have shown that one can identify an empirical quantity, $\mathrm{E}\left(2_{1}^{+}\right)$, that is nearly continuous and in terms of which other quantities, such as $\mathrm{E}\left(4_{1}^{+}\right)$or $\mathrm{S}_{2 n}$, follow simple, compact trajectories with small fluctuations even for large regions of nuclei. We have seen that these observables have distinct anomalies at similar values of $\mathrm{E}\left(2_{1}^{+}\right)$at which phase coexistence in ${ }^{152} \mathrm{Sm}$ has been recently identified. Other observables such as $\mathrm{S}_{2 n}$ may also be used to correlate structural changes. Through a model, such as the IBM (the GCM gives similar results), we have associated the order parameter with a physical quantity, the deformation $\beta_{\text {min }}$, at which the potential has a minimum. In a spherical-deformed transition region in the IBM, $\beta_{\text {min }}$ takes on two characteristic values, zero and then an abrupt change to a near saturation deformation. It appears that nuclear structural evolution in this mass region entails two basic phases (spherical and deformed) rather than a gradual softening (with valence nucleon number) traditionally associated with the onset of deformation in nuclei. Although this view is unconventional, the analysis of the IBM suggests that it is nearly unavoidable and a basic feature of structural evolution. 
We would like to thank F. Iachello, S. Kuyucak, V. J. Emery, S. Shapiro, J. Axe, and M. Straayer for useful discussions of the concept of phase transitions in both finite and infinite systems.

Work supported under DOE Contract Numbers DE-FG02-91ER-40609, DE-FG0291ER-40608 and DE-FG02-88ER-40417. 


\section{References}

[1] H. Eugene Stanley, Introduction to Phase Transitions and Critical Phenomena (Oxford University Press, Oxford, 1971).

[2] Nuclear Data Sheets through June 1998 (Vol.84, No. 2).

[3] R.F. Casten et al, Phys. Rev. C57, R1553 (1998).

[4] F. Iachello, N.V. Zamfir, and R.F. Casten, Phys. Rev. Lett. 81, 1191 (1998).

[5] N.V. Zamfir et al, to be published.

[6] F. Iachello and A. Arima, The Interacting Boson Model, (Cambridge Press, Cambridge, 1987).

[7] R.F. Casten, N.V. Zamfir, and D.S. Brenner, Phys. Rev. Lett. 71, 227 (1993).

[8] R.V. Jolos, P. von Brentano, R.F. Casten, and N.V. Zamfir, Phys. Rev. C51, R2298 (1995).

[9] W.T. Chou, R.F. Casten, N.V. Zamfir, D.S. Brenner, and D. Bucurescu, Nucl. Phys. A580, 33 (1994).

[10] D. Bucurescu, N. Marginean, I. Cata-Danil, M. Ivascu, L. Stroe, and C.A. Ur, Phys.Rev.Lett. B386, 12 (1996); D. Bucurescu and N. Marginean, Phys. Rev. Lett. 79, 31 (1997).

[11] J.L. Wood, K. Heyde, W. Nazarewicz, M. Huyse, and P. van Duppen, Phys. Rep. 215, $101(1992)$

[12] G. Lhersonneau, J.C. Wang, S. Hankonen, P. Dendooven, P. Jones, R. Julin, and J. Äystö, Eur. Phys. J. A2, 25 (1998), G. Lhersonneau et al., Phys. Rev. C56, 2445 (1997)

[13] G. Audi and A.H. Wapstra, Nucl. Phys. A565, 66 (1993).

[14] S. Raman et al. At. Data. Nucl. Data Tables 36, 1 (1987).

[15] G. Gneuss and W. Greiner, Nucl.Phys. A171, 449 (1971).

[16] D.D. Warner and R.F. Casten, Phys. Rev. C28, 1798 (1983).

[17] P.O. Lipas, P. Toivonen, and D.D. Warner, Phys. Lett. 155B, 295 (1985).

[18] N.V. Zamfir and R.F. Casten, Phys. Lett. B341, 1 (1994).

[19] T. Otsuka, A. Arima, and F. Iachello, Nucl. Phys. A309, 1 (1978).

[20] J.N. Ginocchio and M.W. Kirson, Phys. Rev. Lett. 44, 1744 (1980).

[21] A.E.L. Dieperink, O. Scholten, and F. Iachello, Phys. Rev. Lett. 44, 1747 (1980).

[22] O. Scholten, F. Iachello, and A. Arima, Ann. Phys. (NY) 115, 325 (1978). 


\section{Figure Captions}

1: $\mathrm{E}\left(4_{1}^{+}\right)$against neutron number for collective nuclei, that is, nuclei for which $\mathrm{R}_{4 / 2} \equiv$ $\mathrm{E}\left(4_{1}^{+}\right) / \mathrm{E}\left(2_{1}^{+}\right)>2.05$. a) $\left.\mathrm{Sm}, \mathrm{b}\right)$ the $50<\mathrm{Z} \leq 66$ region. Data from ref. [2]

2: $E\left(4_{1}^{+}\right)$plotted against $E\left(2_{1}^{+}\right)$. Panels a-c), show the same data as in fig. 1 for sequentially more elements. Panel d) is an expanded view of the rotational region, with all the data points from panel c) with $E\left(2_{1}^{+}\right)<E_{c}\left(2_{1}^{+}\right)$.

3: (a) Separation energies $S_{2 n}$ as a function of $E\left(2_{1}^{+}\right) . S_{2 n}$ data from ref. [13]. (b) $B\left(E 2: 2_{1}^{+} \rightarrow 0_{1}^{+}\right)$values as a function of $S_{2 n}$. B(E2) data from ref. [14].

4: Classical limit analysis of IBM calculations that reproduce fig. 2c. a) Energy surface as a function of $\xi$ [see eq. (2)] near the critical point for $\mathrm{N}_{B}=10$ and $\chi=-\sqrt{7} / 2$; b) location of the minima, $\beta_{\min }$, as a function of $\xi$, for several values of $\mathrm{N}_{B}$ and $\chi$; and c) The IBM energy $\mathrm{E}(\beta)$ for the $\xi$ values and boson numbers corresponding to the Sm isotopes (see ref. [22]). The minima occur only for $\beta=0$ or large finite values. There is no gradual evolution of $\beta$ from 0 to saturation levels. 


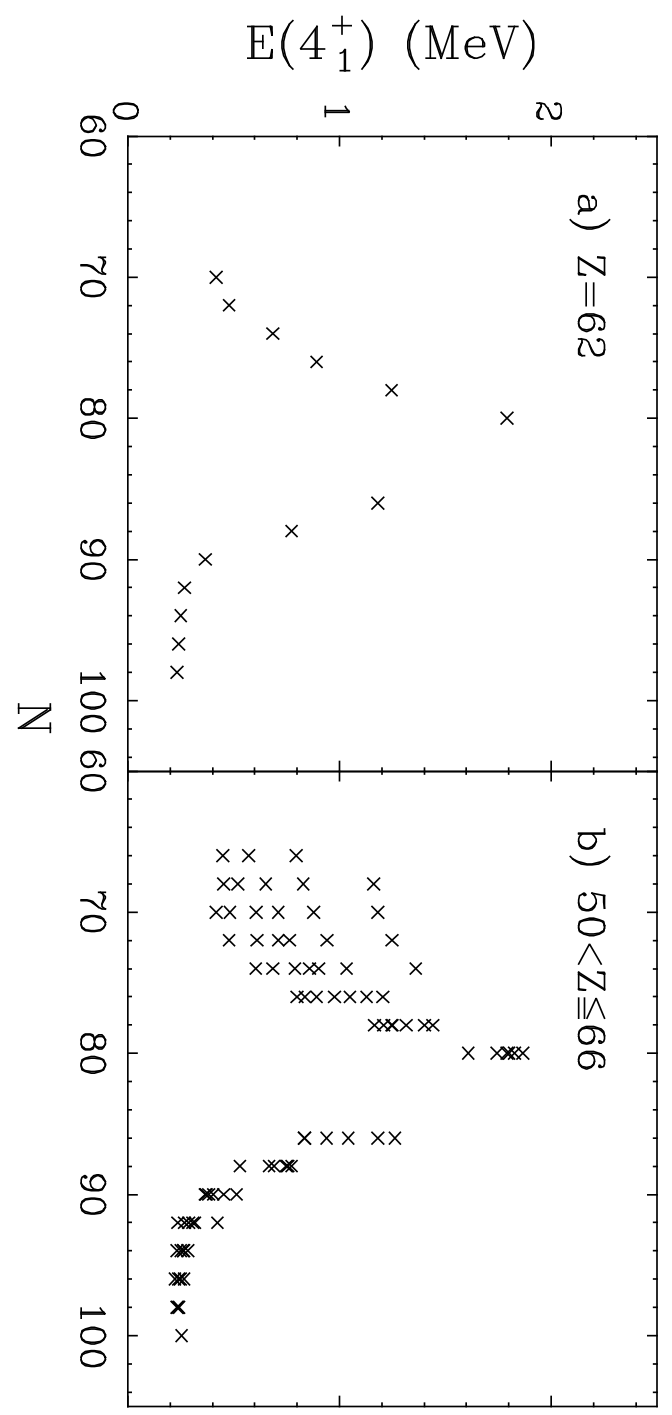




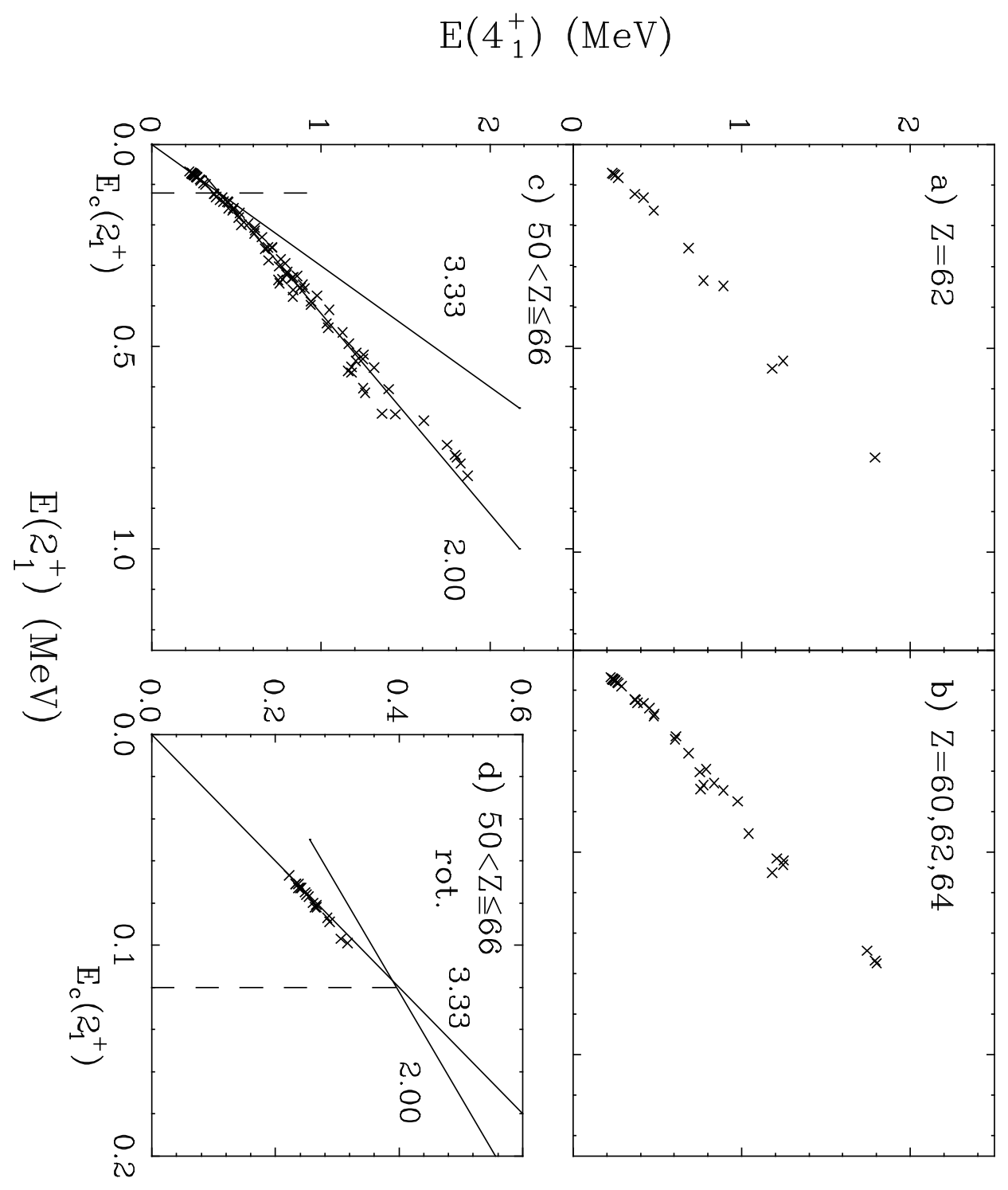




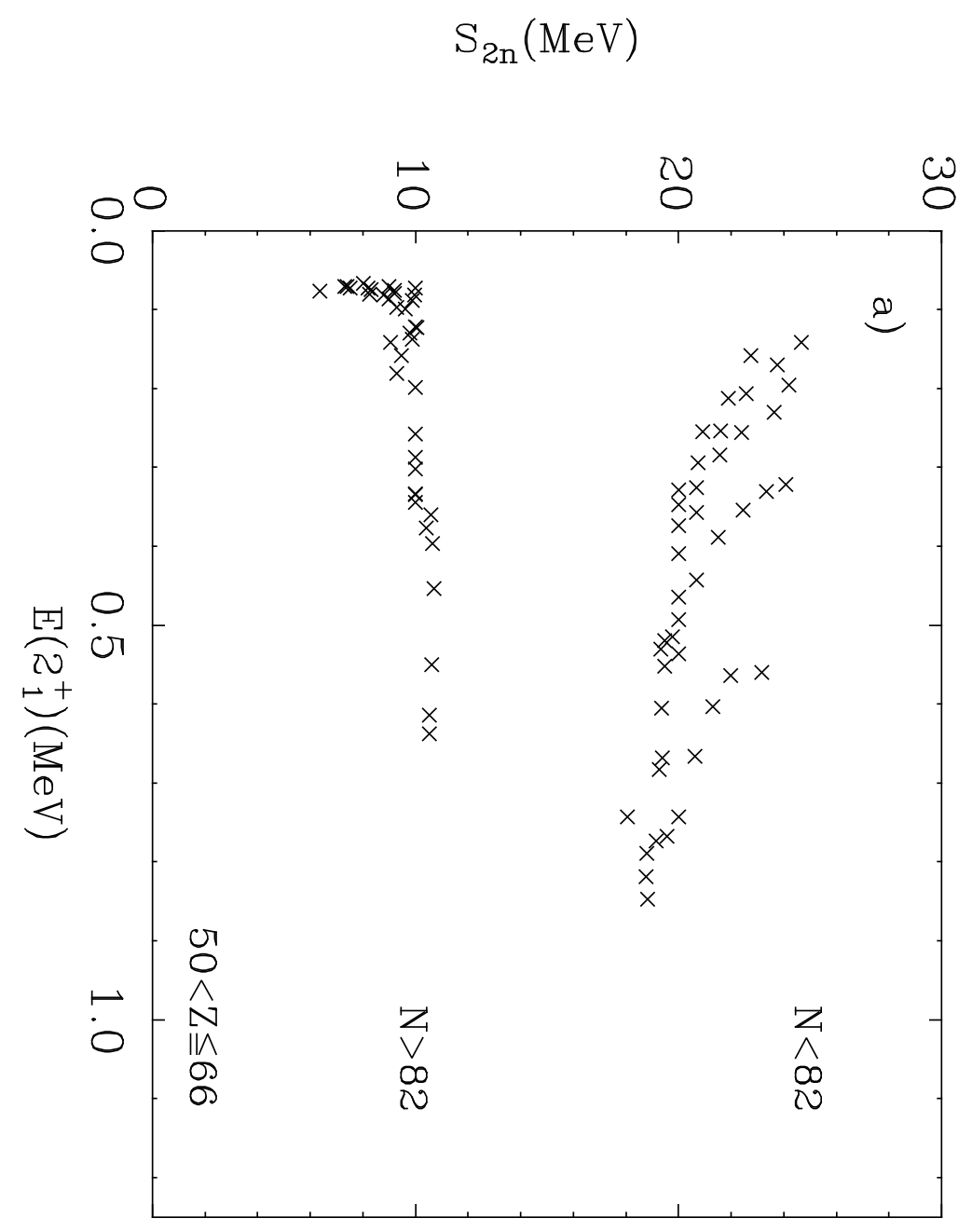




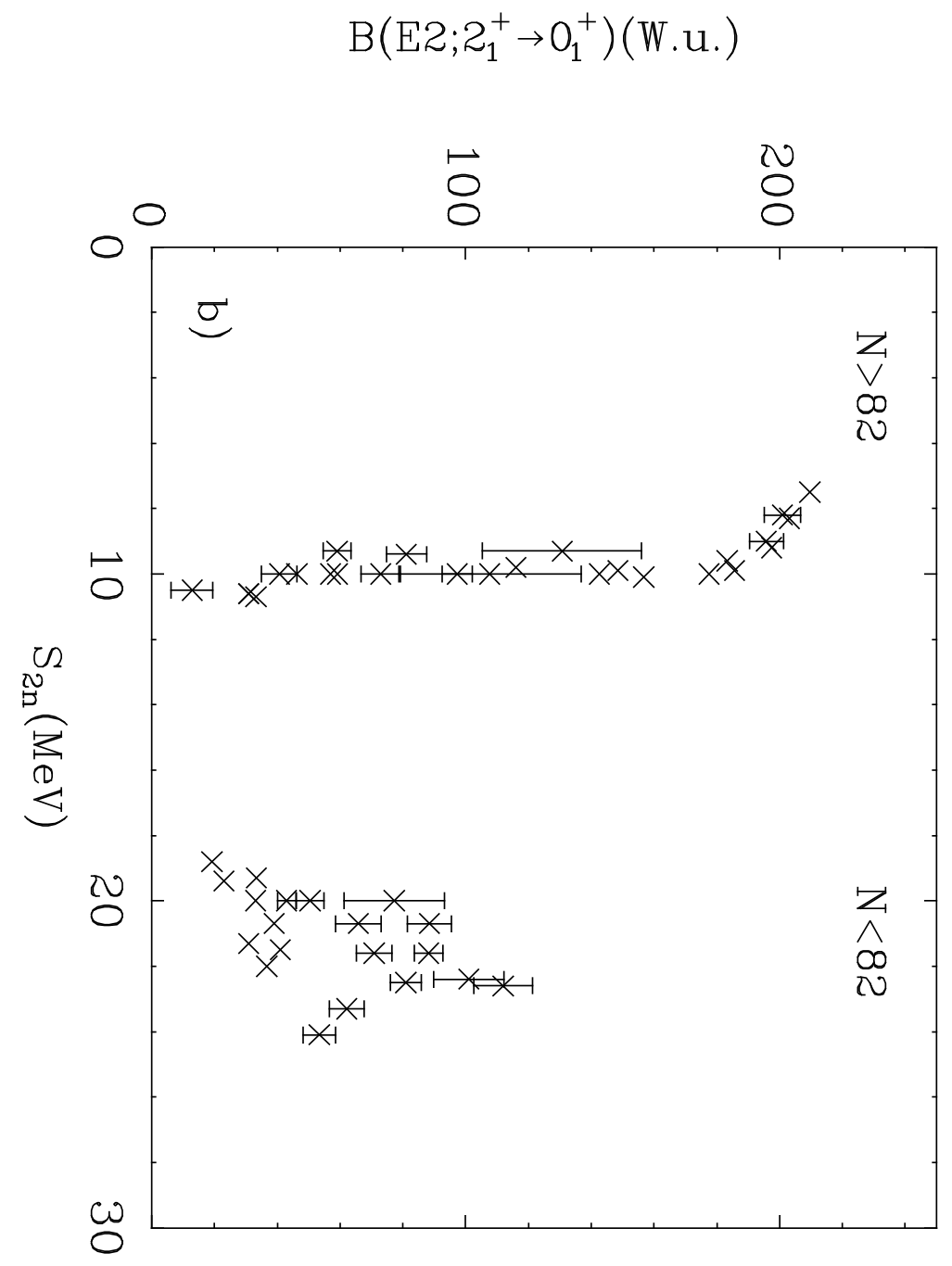




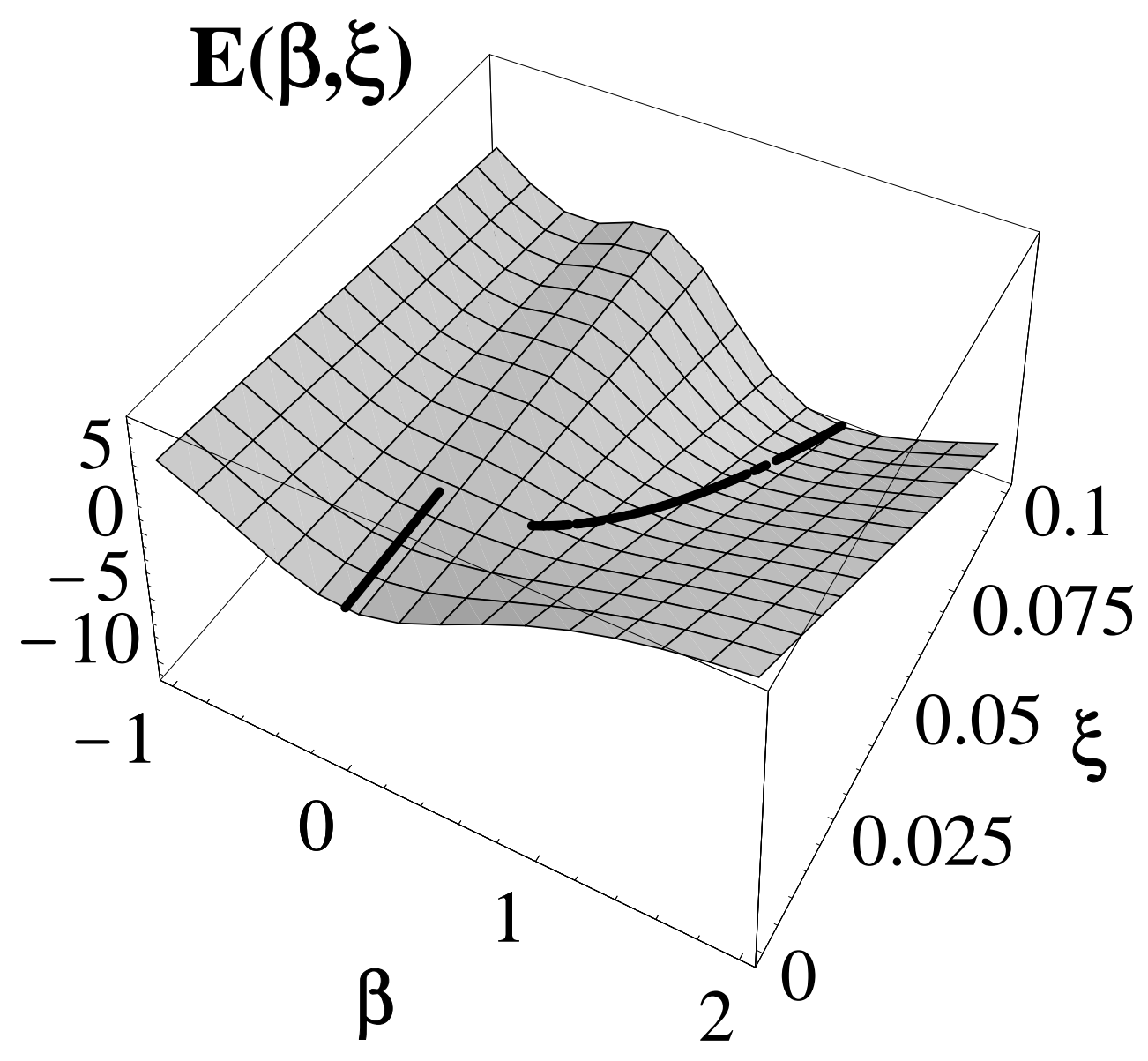




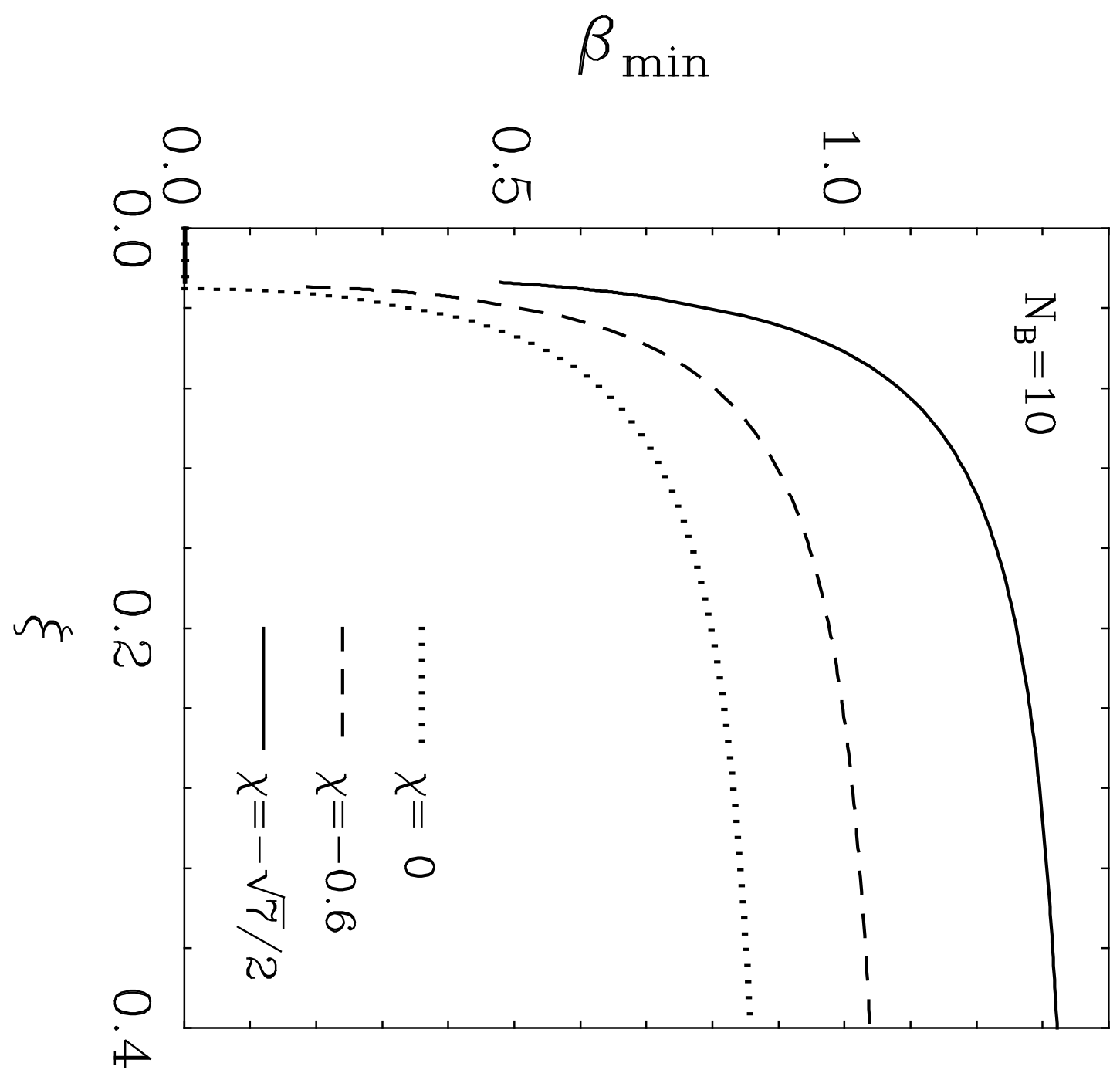




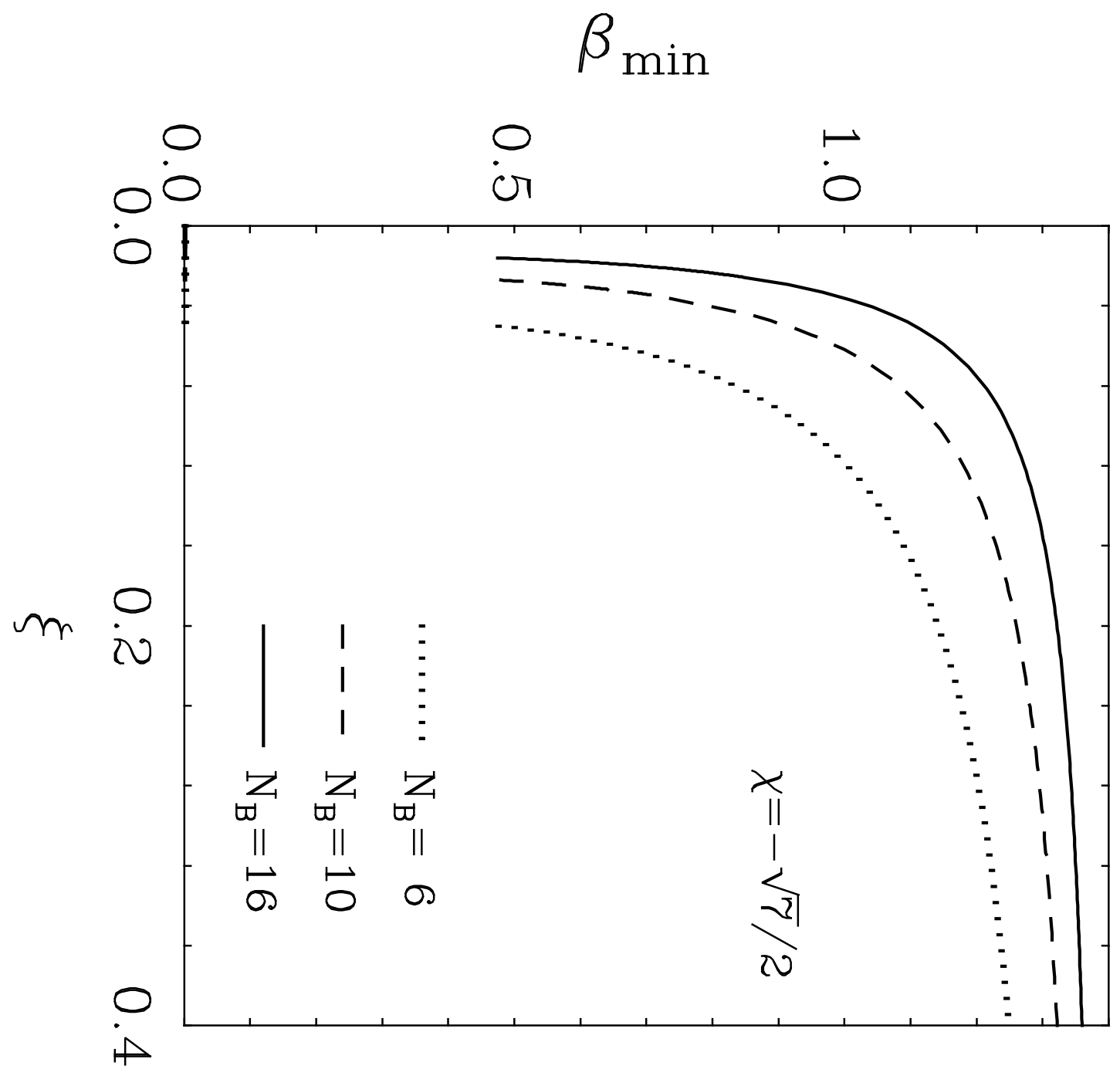




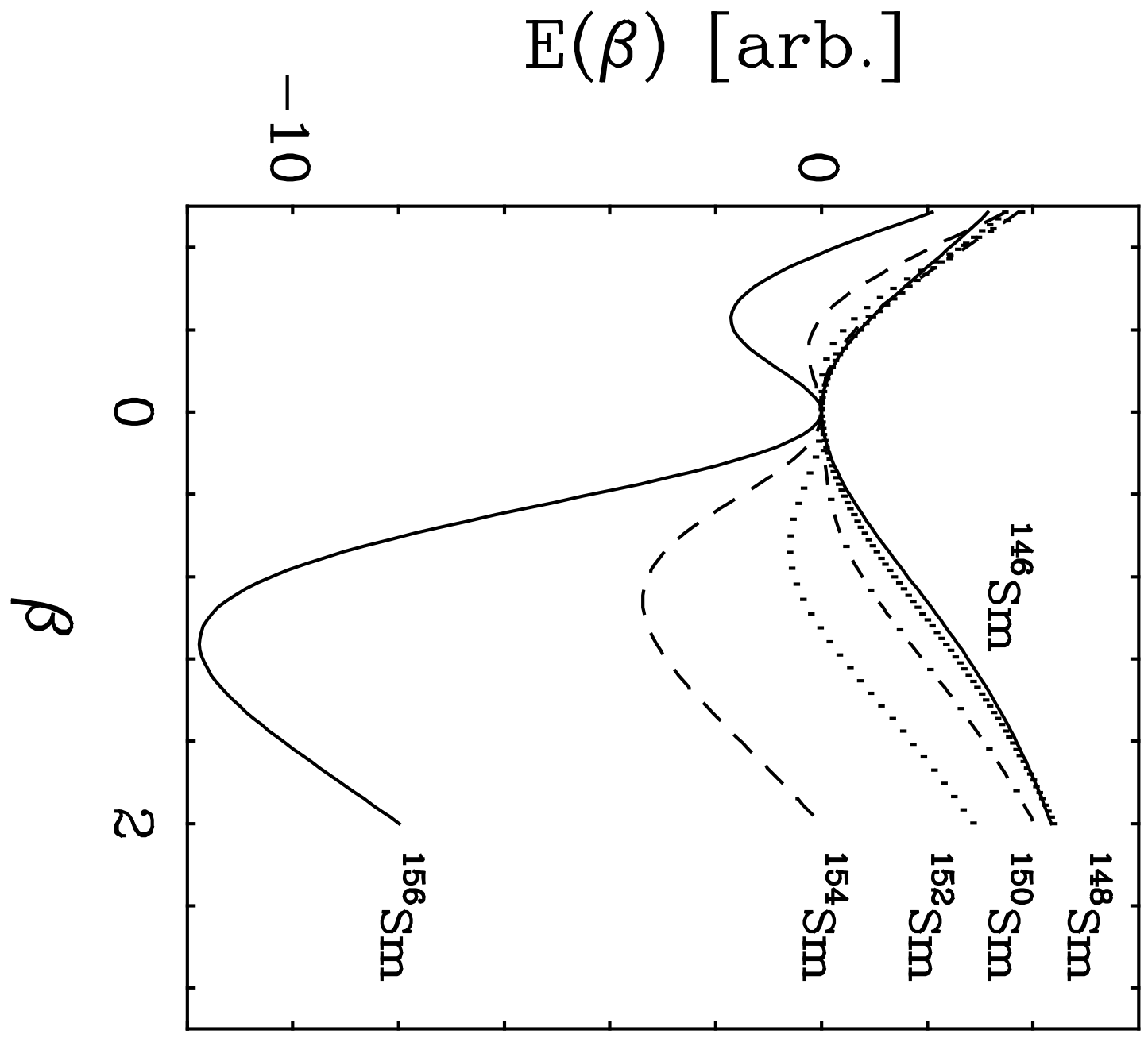

